\title{
New model of abiogenesis
}

\author{
A. IVANOV ${ }^{1}$, V. SEVASTYANOV ${ }^{1}$, A. DolgONOSOV ${ }^{1}$ AND \\ E. GALIMOV ${ }^{1}$
}

${ }^{1}$ Vernadsky Institute of Geochemistry and Analytical Chemistry of Russian Academy of Sciences, Kosygina

street 19, Moscow 119334, Russia (aiva@geokhi.ru)

Understanding the nature of the causes that led to the beginning of the structural self-organization of protobionts is a fundamental question in finding solutions to the problem of abiogenic origin of life. The main difficulties in understanding the question - how this happened, arise due to the fact that after 4 billion years of geological and biological activity of the planet, direct material evidence, the complex process of spontaneous generation of living matter, was not found. But is it possible, with a detailed examination of the geophysical and geochemical features of the situation of the early Earth, to restore the history of the sequence of prebiological events that predetermined the formation of protocellular precursors of the first living organisms? After all, in fact, having embarked on this path, you can trace the order of formation of prebiotic structures reveal the principle of self-organization of primary biological matter, and eventually come to a pristine type of living matter. Probably, the primary living matter required not only special conditions, but also special location that could protect and maintain its existence for a long time, since the aggressive environment of the primitive Earth would not allow primitive life to develop without such protection. This is due to the various kinds of exposure to hard cosmic radiation, as well as the adverse effects of other physical and chemical factors. Therefore, studies of this work were aimed at searching for features of the conditions of the primordial environment, which predetermined the path of the inanimate to the living. The result of this research was a new model of abiogenesis, which sets out the experimental results of testing its real performance [1].

[1] Ivanov A.A., Sevastyanov V.S., Shnykin B.A., Dolgonosov A.A., Krivenko A.P., Priymak S.V., Roslyakova A.S., Galimov E.M. (2019) Experiment in Geosciences Vol. 25 N 1, 27-28 